www.jmscr.igmpublication.org

Index Copernicus Value: 79.54

ISSN (e)-2347-176x ISSN (p) 2455-0450

crossref DOI: https://dx.doi.org/10.18535/jmscr/v7i4.73

\title{
Obstetric and Neonatal Outcome in Higher Parity Pregnant Women in a Tertiary Care Hospital in Kerala
}

\author{
Authors \\ Dr Adma Harshan S. ${ }^{1}$, Dr Anita V. ${ }^{2^{*}}$ \\ ${ }^{1}$ Associate Professor in Obstetrics and Gynaecology, Government College Manjeri \\ ${ }^{2}$ Associate Professor in Obstetrics and Gynaecology, Government College Trivandrum \\ *Corresponding Author \\ Dr Anita V \\ Email: anitaunnikrishnan@gmail.com
}

\begin{abstract}
Introduction: Higher parity pregnancy is common in developing countries and is considered as a high risk factor for maternal and perinatal complications. The objective of the study was to compare the obstetric and neonatal outcomes in higher parity with that of lower parity in our hospital. Retrospective comparative study was done in a tertiary care hospital in Government Medical College Manjeri, Malappuram, Kerala, India from April to June 2017.Data from labour room records was collected from 131 lower parity (P1) pregnant women and 259 higher parity women (P2 and higher) who came and gave birth in the hospital .The obstetric and neonatal outcomes of both groups were compared using appropriate statistical tools. $P<0.05$ was taken as significant.

Results: A total of 390 women participated in the study. Higher parity women had higher incidence of anaemia, hypertensive disorders, intrauterine growth restriction (IUGR) and more of meconium stained amniotic fluid. Occurrence of Diabetes both gestational and pregestational and incidence of atonic postpartum haemorrhage, however, was paradoxically low.

Conclusion: Higher parity and its associated complications still occur frequently. However, limiting parity might improve both maternal and perinatal morbidity and mortality. Health facility based family planning awareness should be instituted. Adequate antenatal surveillance, optimal intrapartum and postpartum care will further reduce the complications.
\end{abstract}

Keywords: Higher parity, multiparity, Anaemia, hypertensive disorders.

\section{Introduction}

High risk pregnancy is defined as one in which mother, foetus or newborn are at increased risk of morbidity or mortality before, at or after birth. It has also been shown that the best obstetric outcome is often seen in women who are para one two and three. This risk to mother and child is high in first pregnancy $(\mathrm{P} 0)$, then the risk declines in $2^{\text {nd }}, 3^{\text {rd }}$ and then slowly rises with increasing parity and by sixth pregnancy, risk exceeds those of $1^{\text {st }}$ and after that rises steeply with each pregnancy ${ }^{1}$. Keeping this in mind, a study was planned excluding the primigravidas and including para 1,2,3 and further higher parity pregnant women. Being practising obstetricians, the authors were aware that the $2^{\text {nd }}$ gravidas ( $\mathrm{P}$ 1) were the ones who would be 
managed with maximum ease and minimum risk. Further higher parity women (P 2 and higher) had apparently more risk factors and complications compared to para 1. FIGO defines grand multipara as women who have delivered 5 to 9 fetuses whereas women who have delivered 10 or more times are considered to be great grand multiparas.

Incidence of grand multipara is slowly declining in most countries since two generations due to better socioeconomic and educational status, better contraceptive services and utilisation and understanding of limitation of Earth's resources. In developed countries, grand multiparity is becoming rare with incidence of $1-4 \%$ of all births, while in developing countries, they have been considered to be at higher risk of developing antenatal complications. Complications seen in grand multiparous women are anaemia, hypertension, gestational diabetis mellitus, placental abruption placenta praevia, preterm labour, malpresentation and feto pelvic disproportion. Other complications include uterine inertia, uterine rupture, fetal macrosomia, postpartum haemorrhage and operative deliveries with its consequent risk of maternal morbidity and mortality.

Socio economic factors play a very important part; majority of these patients are poor with inadequate access to modern perinatal care coupled with increased maternal age. They tend to feed their numerous children at the expense of their own nutrition, thus are prone to malnutrition. They are too busy to attend to their health and due to rapid succession of pregnancies and lactation, there is subsequent iron and calcium depletion. Poor stores coupled with suboptimal care will subsequently lead to antenatal and perinatal complications.

In India, incidence of grand multiparity is coming down with better family planning interventions to grand multiparity and its complications. Although grand multiparity has long been considered to be associated with increased maternal and fetal complications, recent studies indicated that with proper perinatal care, women with high parity rates are no longer at high risk. A study was undertaken in Government Medical College, Manjeri,
Malappuram to understand and compare the obstetric outcome in higher parity women (P 2 and higher) in comparison to lower parity (P 1). Number of Para 3 (women who have given birth to 3 children) and para 4 onwards are rapidly declining in most part of state of Kerala except in Northern Kerala mainly because of religious compulsions. But the incidence of grand multiparity (para 4 and more) is becoming less and less even in these areas due to improving education and socio economic status and utilisation of contraceptive services. Hence, para 1 (lower parity) are being compared with para $=2$ or $>$ (higher parity) in this study to assess the obstetric outcome and complications in higher parity women in Government Medical College Manjeri. The study will help in increasing awareness regarding complications and suggest ways to improve care of patients and also improve maternal quality of life and fetal survival.

\section{Aim of the study}

To compare the obstetric and neonatal outcome in pregnant women who are para one (lower parity) and women having parity more than one (higher parity). The women will be compared for socio economic characteristics, education, occurrence of pregnancy complications and perinatal outcome

\section{Operational definitions}

Lower parity- Pregnant Women who have already delivered one child

Higher parity - Pregnant women who have delivered two or more children

Hypothesis- Obstetric complications are more in higher parity than lower parity.

\section{Materials and methods}

This study was conducted in Obstetrics and Gynaecology department at Government Medical College Manjeri, Malappuram, Kerala State, India. This is located in the north part of Kerala and dominant religious community in this part is Muslim. Average monthly delivery in this hospital is around 450 .This hospital also has more number of multiparous and higher parity women delivering 
in this hospital as compared to other regions of the state. Hence this topic was selected for the purpose of the study. Duration of the study was three months which extended from May 2017 to July 2017. The data was retrospectively collected from the obstetric registers from labour rooms. All multiparous women who delivered during this period were included in the study. Sample selection was done according to following inclusion and exclusion criteria. Inclusion criteria was all pregnant multiparous women. Exclusion criteria was primigravidas. The study design was comparative and retrospective. Information regarding women of lower and higher parity were collected retrospectively from the labour records. A total of 131 women with parity 1 (lower parity) and 259 women with parity of 2 and more (higher parity) were admitted during the study period and their obstetric and perinatal outcomes were compared. Study variables included age, religion, education, pregnancy complication like hypertension, anaemia, diabetes mellitus, malpresentation, prematurity, mode of delivery, occurrence of post partum haemorrhage $(\mathrm{PPH})$, neonatal intensive care admissions (NICU) were compared. Anaemia was taken as haemoglobin less than $11 \mathrm{~g} \%$. Pregnancy induced hypertension (PIH) was defined as blood pressure $>140 / 90 \mathrm{mmHg}$ after 20 weeks of pregnancy with or without proteinuria taken 6 hours apart. Bleeding from genital tract after 24 weeks was taken as ante partum haemorrhage (APH). Malpresentation was defined as presenting part of foetus is other than cephalic in relation to maternal pelvis. Preterm labour was defined as onset of labour before 37 completed weeks of gestation. Mode of delivery was also recorded. After delivery, patients are monitored for 24 hours for primary $\mathrm{PPH}$ which is taken as blood loss more than $500 \mathrm{ml}$ in vaginal delivery and more than $1000 \mathrm{ml}$ in Caesarean section. Neonates were also followed up for complications like low birth weight (LBW), meconium aspiration, NICU admissions. Birth weight of $<2.5 \mathrm{~kg}$ is taken as LBW. Data was collected through special proforma. Neonatal follow up was also recorded in the same proforma. Data analysis was done by appropriate statistical tools. Percentages and frequencies were calculated for presentation of categorical variables. Chi square test was applied to compare variables between lower and higher parity groups. $p$ value of $<0.05$ was taken as statistically significant.

\section{Results}

Background characteristics of sample

Table Distribution of age based on parity

\begin{tabular}{|l|c|c|c|c|}
\hline \multirow{2}{*}{ Age } & \multicolumn{2}{|c|}{ P1 } & \multicolumn{2}{c|}{ P2 \& above } \\
\cline { 2 - 5 } & Count & Percent & Count & Percent \\
\hline$<=20$ & 11 & 8.4 & 4 & 1.5 \\
\hline $21-25$ & 72 & 55.0 & 52 & 20.1 \\
\hline $26-30$ & 37 & 28.2 & 110 & 42.5 \\
\hline $31-35$ & 6 & 4.6 & 73 & 28.2 \\
\hline$>35$ & 5 & 3.8 & 20 & 7.7 \\
\hline Mean \pm SD & \multicolumn{3}{|c|}{$25 \pm 4.2$} & \multicolumn{2}{c|}{$29 \pm 4.3$} \\
\hline
\end{tabular}

More of higher women were in the age group 26-30 years.

Table Distribution of religion based on parity

\begin{tabular}{|l|c|c|c|c|}
\hline \multirow{2}{*}{ Religion } & \multicolumn{2}{|c|}{ P1 } & \multicolumn{2}{c|}{ P2 \& above } \\
\cline { 2 - 5 } & Count & Percent & Count & Percent \\
\hline Hindu & 35 & 26.7 & 45 & 17.4 \\
\hline Christian & 0 & 0.0 & 1 & 0.4 \\
\hline Muslim & 96 & 73.3 & 213 & 82.2 \\
\hline
\end{tabular}

Majority religion in both groups was Muslim 
Table Comparison of educational status based on parity

\begin{tabular}{|l|c|c|c|c|c|c|}
\hline \multirow{2}{*}{ Educational status } & \multicolumn{2}{|c|}{ P1 } & \multicolumn{2}{c|}{ P2 \& above } & \multirow{2}{*}{$\chi^{2}$} & \multirow{2}{*}{$\mathrm{p}$} \\
\cline { 2 - 5 } & Count & Percent & Count & Percent & & \\
\hline Below 10th & 19 & 14.5 & 64 & 24.7 & & \\
\hline 10th & 42 & 32.1 & 120 & 46.3 & \multirow{2}{*}{23.} & $\mathrm{p}<0.01$ \\
\hline Higher secondary & 52 & 39.7 & 60 & 23.2 & 3 & \\
\hline Above Hr. Secondary & 18 & 13.7 & 15 & 5.8 & & \\
\hline
\end{tabular}

Higher parity pregnant women had poor education.

\section{Comparison of maternal complication based on Parity}

Table Comparison of maternal complication based on Parity

\begin{tabular}{|c|c|c|c|c|c|c|c|}
\hline \multirow{2}{*}{\multicolumn{2}{|c|}{$\begin{array}{c}\text { Maternal } \\
\text { complication }\end{array}$}} & \multicolumn{2}{|c|}{ P1 } & \multicolumn{2}{|c|}{ P2 \& above } & \multirow{2}{*}{$\chi^{2}$} & \multirow{2}{*}{$\mathrm{p}$} \\
\hline & & \multirow{2}{*}{$\frac{\text { Count }}{29}$} & \multirow{2}{*}{$\frac{\text { Percent }}{22.1}$} & \multirow{2}{*}{$\frac{\text { Count }}{67}$} & \multirow{2}{*}{$\frac{\text { Percent }}{25.9}$} & & \\
\hline \multirow{2}{*}{ Anemia } & Present & & & & & \multirow{2}{*}{0.65} & \multirow{2}{*}{0.419} \\
\hline & Absent & 102 & 77.9 & 192 & 74.1 & & \\
\hline \multirow{2}{*}{ HTN } & Present & 10 & 7.6 & 21 & 8.1 & \multirow{2}{*}{0.03} & \multirow{2}{*}{0.870} \\
\hline & Absent & 121 & 92.4 & 238 & 91.9 & & \\
\hline \multirow{2}{*}{ GDM/DM } & Present & 12 & 9.2 & 17 & 6.6 & \multirow{2}{*}{0.85} & \multirow{2}{*}{0.356} \\
\hline & Absent & 119 & 90.8 & 242 & 93.4 & & \\
\hline \multirow{2}{*}{ Preterm } & Present & 6 & 4.6 & 8 & 3.1 & \multirow{2}{*}{0.56} & \multirow{2}{*}{0.455} \\
\hline & Absent & 125 & 95.4 & 251 & 96.9 & & \\
\hline \multirow{2}{*}{ PP/AP } & Present & 0 & 0.0 & 1 & 0.4 & \multirow{2}{*}{0.51} & \multirow{2}{*}{0.476} \\
\hline & Absent & 131 & 100.0 & 258 & 99.6 & & \\
\hline \multirow{2}{*}{ Twins } & Present & 3 & 2.3 & 3 & 1.2 & \multirow{2}{*}{0.74} & \multirow{2}{*}{0.391} \\
\hline & Absent & 128 & 97.7 & 256 & 98.8 & & \\
\hline \multirow{2}{*}{ Mal pres } & Present & 3 & 2.3 & 6 & 2.3 & \multirow{2}{*}{0} & \multirow{2}{*}{0.987} \\
\hline & Absent & 128 & 97.7 & 253 & 97.7 & & \\
\hline \multirow{2}{*}{ PROM } & Present & 7 & 5.3 & 11 & 4.2 & \multirow{2}{*}{0.24} & \multirow{2}{*}{0.626} \\
\hline & Absent & 124 & 94.7 & 248 & 95.8 & & \\
\hline
\end{tabular}

Hypertensive disorders were significantly higher in higher parity, also anaemia. But Diabetes, prematurity and twins showed a lower incidence.

Table Comparison of maternal complication based on Parity

\begin{tabular}{|c|c|c|c|c|c|c|c|}
\hline \multirow{2}{*}{\multicolumn{2}{|c|}{ Maternal complication }} & \multicolumn{2}{|c|}{ P1 } & \multicolumn{2}{|c|}{ P2 \& above } & \multirow{2}{*}{$\chi^{2}$} & \multirow[b]{2}{*}{$\mathrm{p}$} \\
\hline & & \multirow{2}{*}{$\frac{\text { Count }}{3}$} & \multirow{2}{*}{$\frac{\text { Percent }}{2.3}$} & \multirow{2}{*}{$\frac{\text { Count }}{8}$} & \multirow{2}{*}{$\frac{\text { Percent }}{3.1}$} & & \\
\hline \multirow{2}{*}{ IUGR } & Present & & & & & \multirow{2}{*}{0.2} & \multirow{2}{*}{0.653} \\
\hline & Absent & 128 & 97.7 & 251 & 96.9 & & \\
\hline \multirow{2}{*}{$\mathrm{PPH}-\mathrm{a} / \mathrm{t}$} & Present & 3 & 2.3 & 2 & 0.8 & \multirow[t]{2}{*}{1.58} & \multirow{2}{*}{0.208} \\
\hline & Absent & 128 & 97.7 & 257 & 99.2 & & \\
\hline \multirow{2}{*}{ Episio } & Present & 28 & 21.4 & 21 & 8.1 & \multirow{2}{*}{13.94} & \multirow{2}{*}{$\mathrm{p}<0.01$} \\
\hline & Absent & 103 & 78.6 & 238 & 91.9 & & \\
\hline \multirow{3}{*}{ Peri tear } & Normal & 89 & 67.9 & 167 & 64.5 & \multirow{3}{*}{2.28} & \multirow{3}{*}{0.320} \\
\hline & 1st degree & 42 & 32.1 & 88 & 34.0 & & \\
\hline & 2nd degree & 0 & 0.0 & 4 & 1.5 & & \\
\hline \multirow{2}{*}{ B1. Transf } & Present & 2 & 1.5 & 0 & 0.0 & \multirow{2}{*}{$3.97 *$} & \multirow{2}{*}{0.046} \\
\hline & Absent & 129 & 98.5 & 259 & 100.0 & & \\
\hline \multirow{2}{*}{ Induced } & Present & 23 & 17.6 & 40 & 15.4 & \multirow{2}{*}{0.29} & \multirow{2}{*}{0.592} \\
\hline & Absent & 108 & 82.4 & 219 & 84.6 & & \\
\hline \multirow{2}{*}{ Past Date } & Present & 4 & 3.1 & 5 & 1.9 & \multirow{2}{*}{0.49} & \multirow{2}{*}{0.485} \\
\hline & Absent & 127 & 96.9 & 254 & 98.1 & & \\
\hline \multirow{2}{*}{ Mode of delivery } & Normal & 87 & 66.4 & 187 & 72.5 & \multirow[t]{2}{*}{1.54} & \multirow{2}{*}{0.215} \\
\hline & LSCS & 44 & 33.6 & 71 & 27.5 & & \\
\hline
\end{tabular}

*Significant at 0.05 level PPH was lower but IUGR was higher in higher parity. More number of vaginal deliveries were reported in higher parity. 
Comparison of fetal complication based on Parity

Table Comparison of fetal complication based on Parity

\begin{tabular}{|c|c|c|c|c|c|c|c|}
\hline \multirow{2}{*}{\multicolumn{2}{|c|}{ Fetal complication }} & \multicolumn{2}{|c|}{ P1 } & \multicolumn{2}{|c|}{ P2 \& above } & \multirow{2}{*}{$\chi^{2}$} & \multirow[b]{2}{*}{$\mathrm{p}$} \\
\hline & & Count & Percent & Count & Percent & & \\
\hline \multirow{3}{*}{ Weight } & $<2.5$ & 27 & 20.6 & 57 & 22.0 & \multirow{3}{*}{1.32} & \multirow{3}{*}{0.516} \\
\hline & $2.5-3.5$ & 93 & 71.0 & 188 & 72.6 & & \\
\hline & $>3.5$ & 11 & 8.4 & 14 & 5.4 & & \\
\hline \multirow{2}{*}{ Meco } & Present & 3 & 2.3 & 8 & 3.1 & \multirow{2}{*}{0.2} & \multirow{2}{*}{0.653} \\
\hline & Absent & 128 & 97.7 & 251 & 96.9 & & \\
\hline \multirow{2}{*}{ Congenital ano } & Present & 0 & 0.0 & 3 & 1.2 & \multirow{2}{*}{1.53} & \multirow{2}{*}{0.216} \\
\hline & Absent & 131 & 100.0 & 256 & 98.8 & & \\
\hline \multirow{3}{*}{ Sh DYS } & Present & 0 & 0.0 & 0 & 0.0 & \multirow{3}{*}{1.29} & \multirow{3}{*}{0.256} \\
\hline & Absent & 131 & 100.0 & 259 & 100.0 & & \\
\hline & Present & 3 & 2.3 & 12 & 4.6 & & \\
\hline
\end{tabular}

Background characteristics of sample Meconium stained amniotic fluid and shoulder dystocia slightly higher in higher parity.

Fig. Distribution of age based on parity

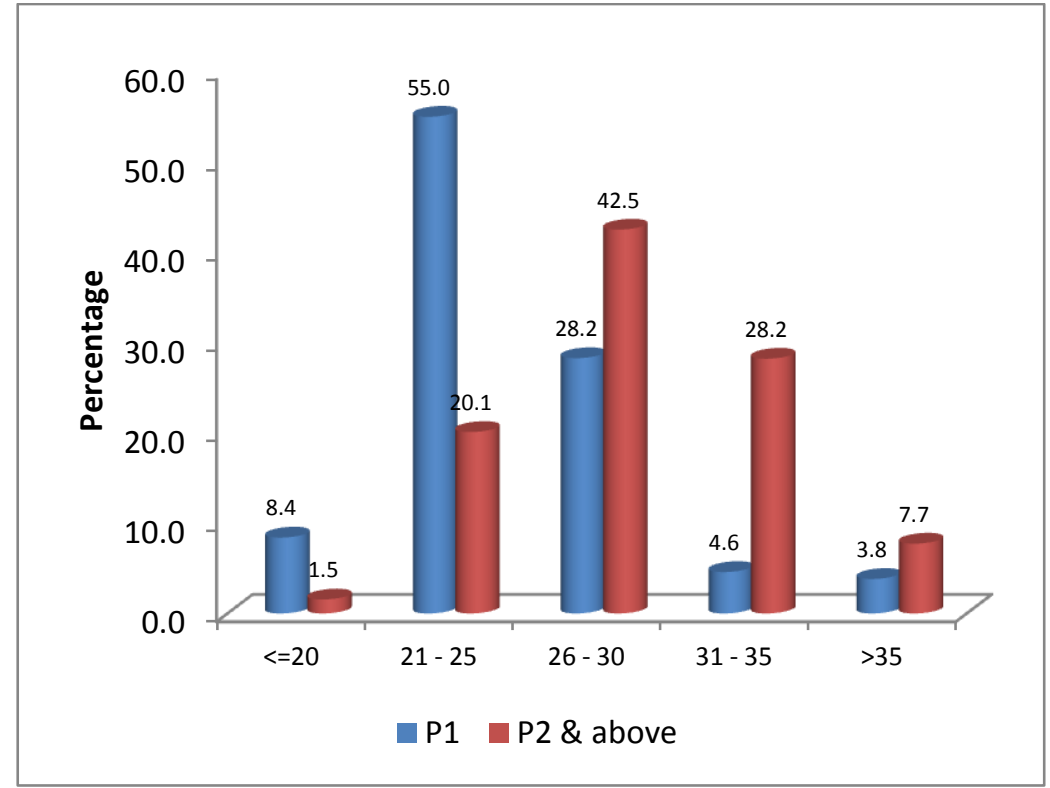

Fig. Distribution of religion based on parity

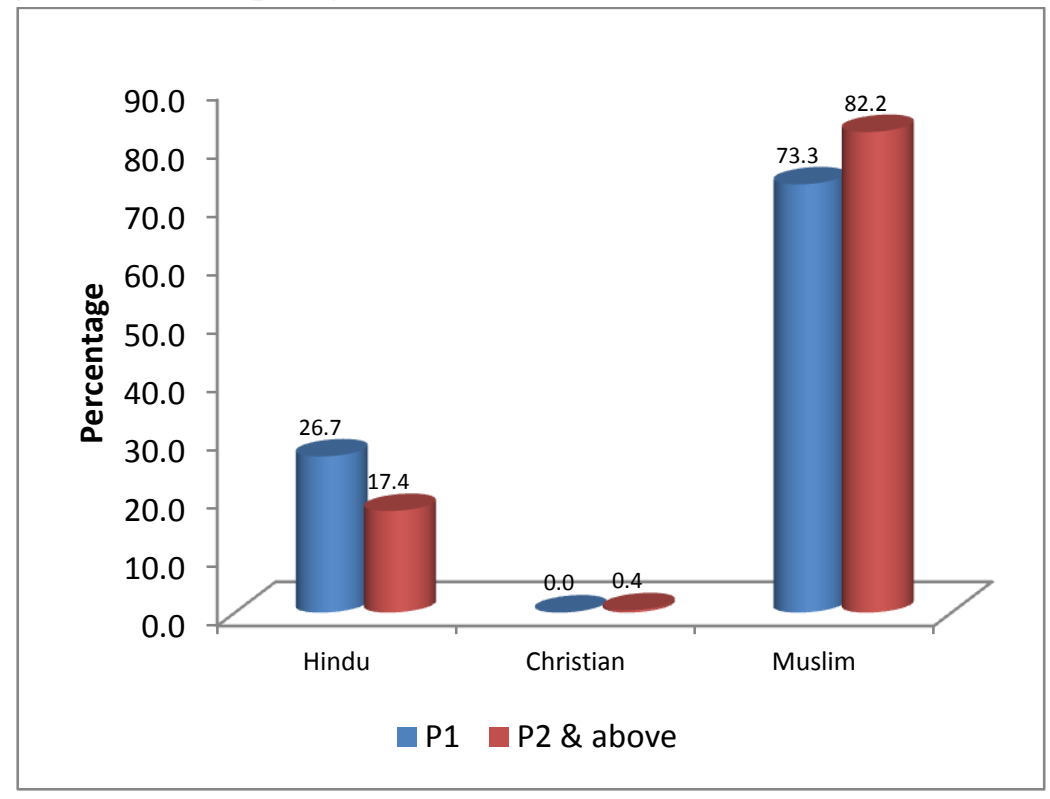


Fig. Comparison of educational status based on parity

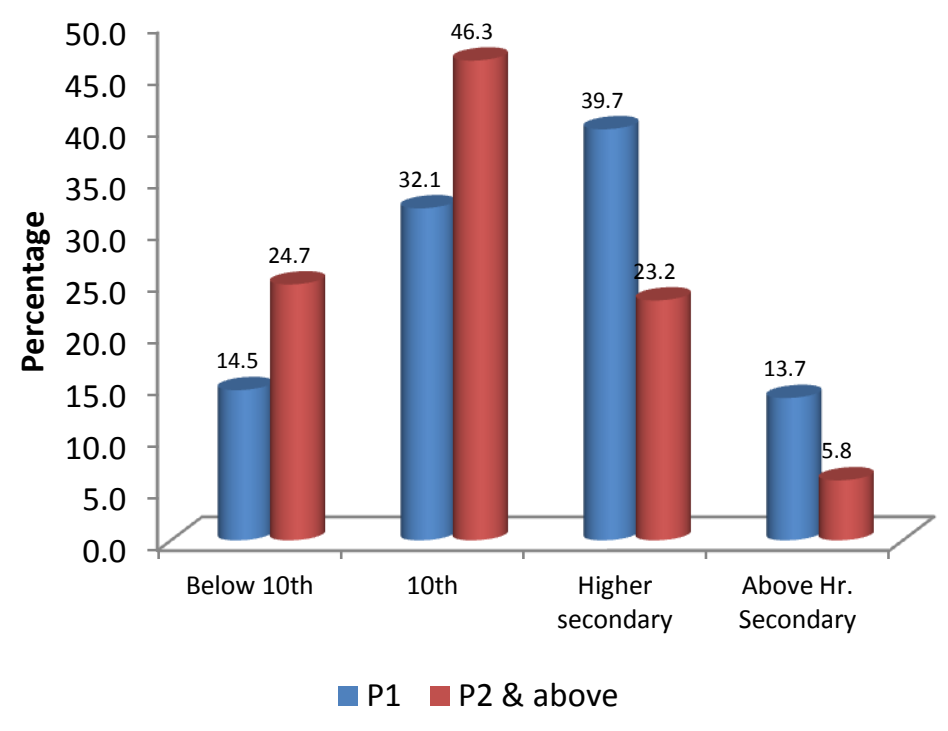

\section{Comparison of maternal complication based on Parity}

Fig. Comparison of maternal complication based on Parity

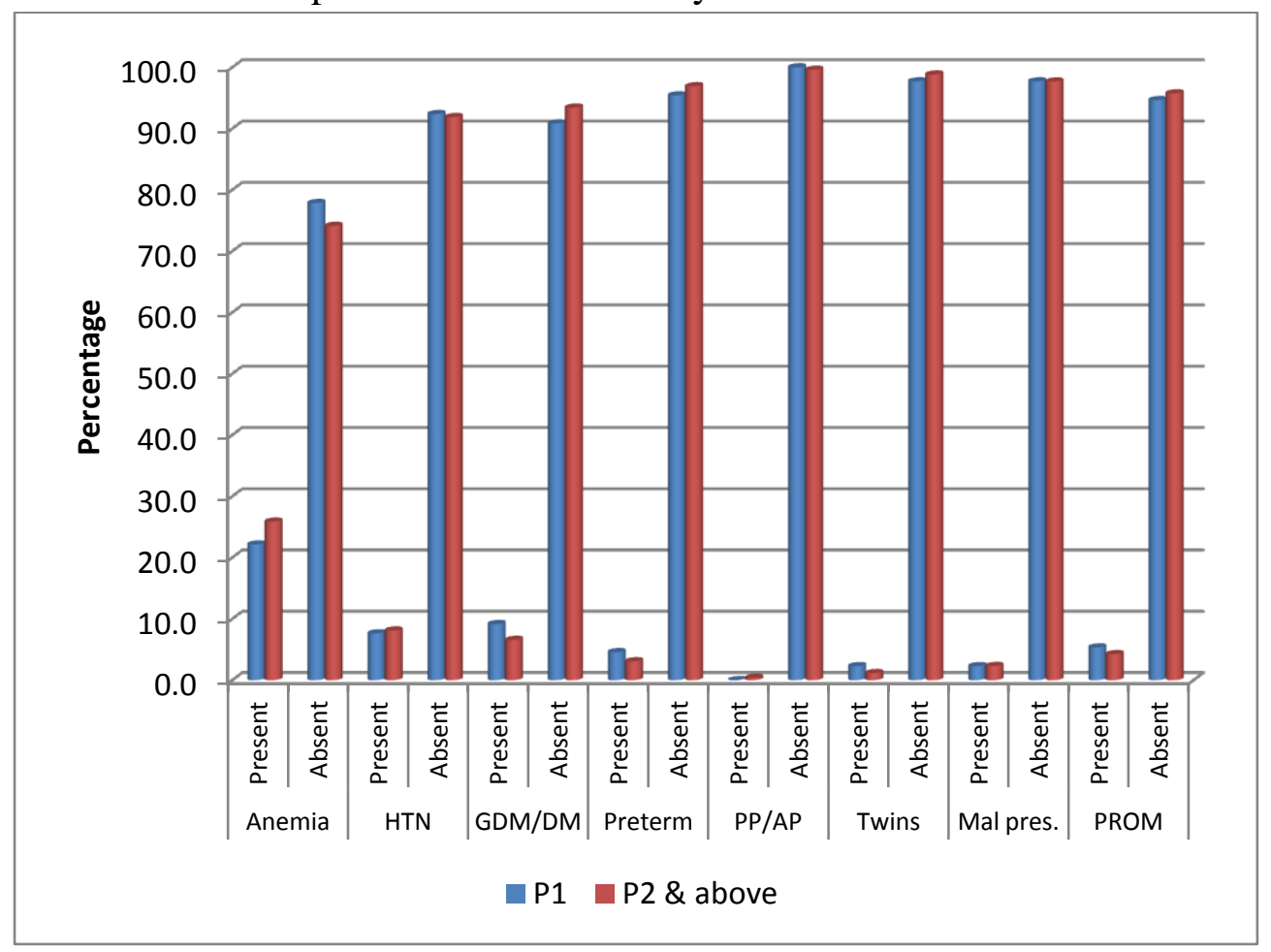




\section{JMSCR Vol||07||Issue||04||Page 424-432||April}

Fig. Comparison of maternal complication based on Parity

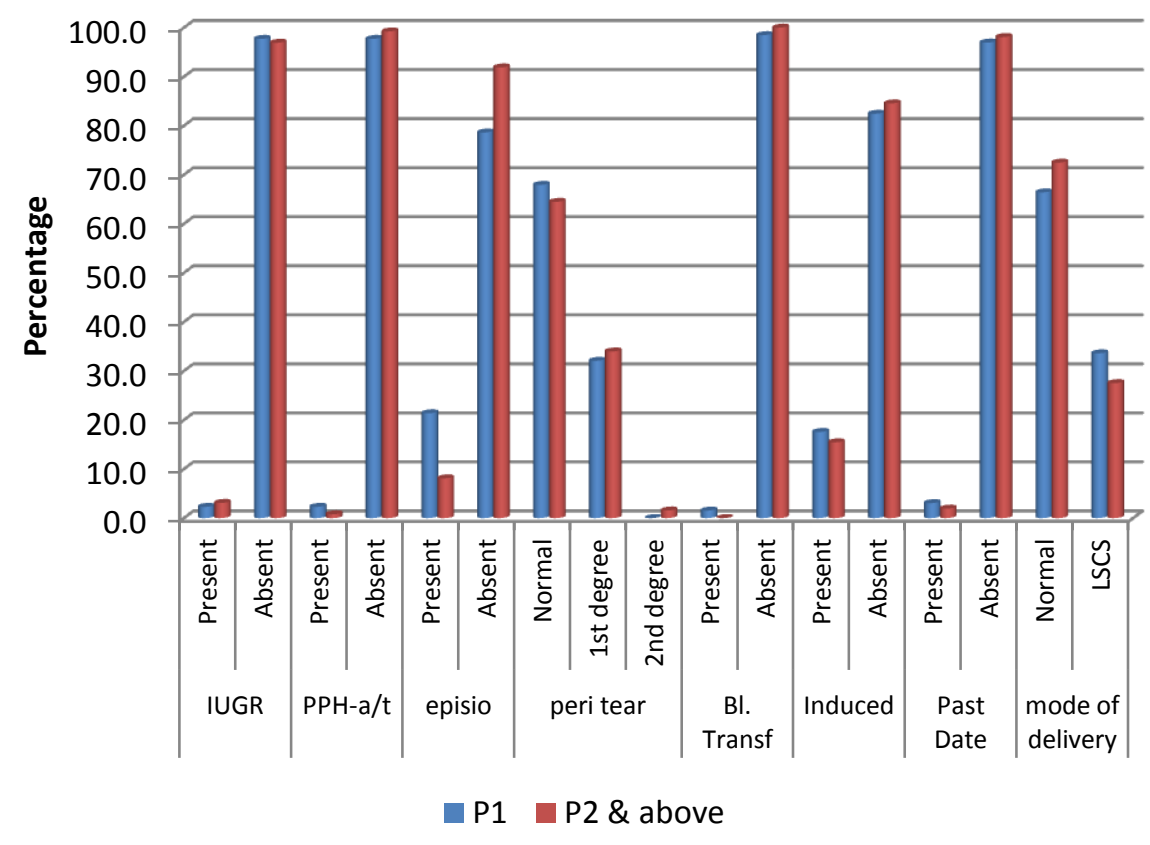

Fig. Comparison of fetal complication based on Parity

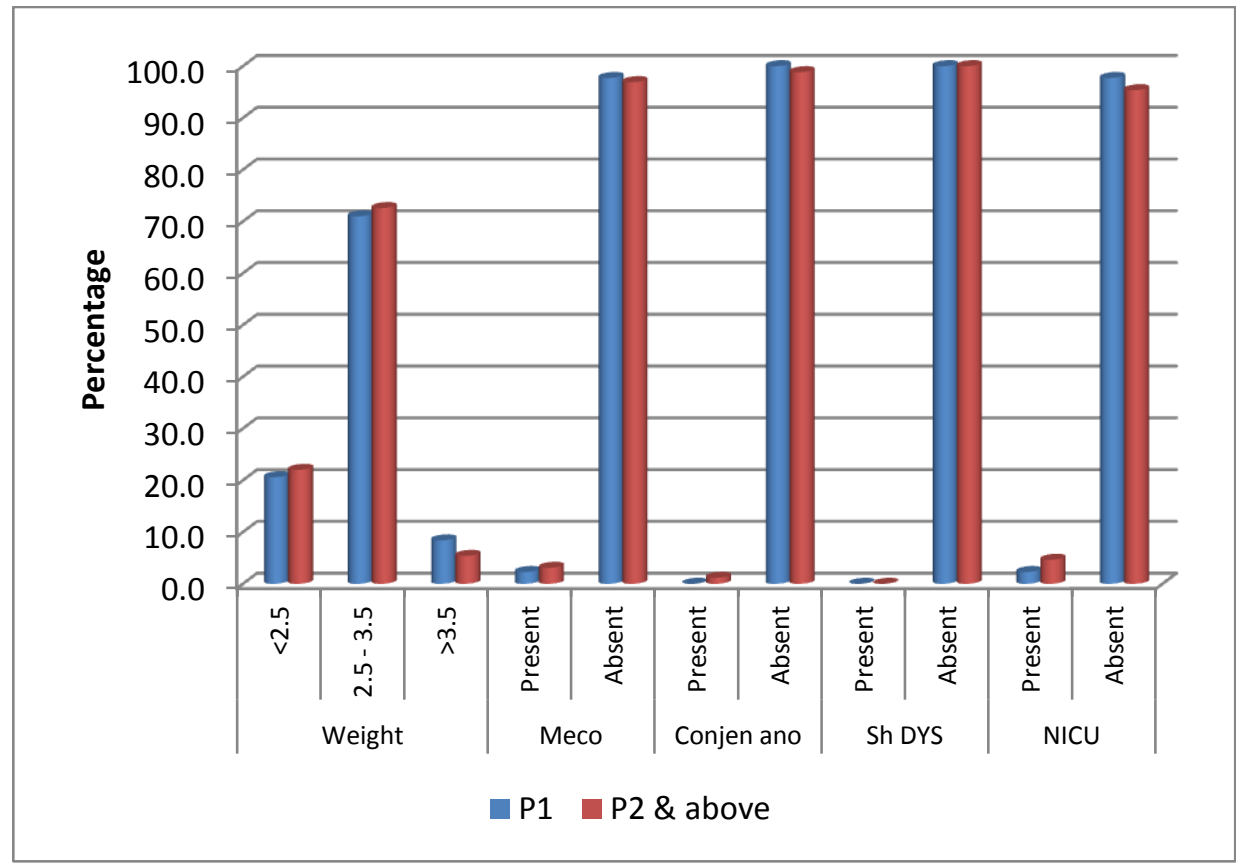

\section{Discussion}

The peak age incidence of higher parity was 26-30 years similar to that of Maiduguri ${ }^{[2]}$ but lower than that in $\operatorname{Lagos}^{[3]}$ and Calabar, Nigeria ${ }^{[1]}$. This shows that most multiparous women continue to be pregnant until age related subfertility sets in. It is interesting to note $1.5 \%$ of higher parity recorded are of age $<=20$ years; reflecting possible early age at marriage and childbearing in our circumstances.
Majority religion in both higher and lower parity groups was found to be Muslim. In world over, multiparity is more associated with Islamic religion. This is not unexpected as the culture of polygamous marriage and large family size is common among Muslims. Low educational status was significantly associated with higher parity in our study. Similar findings were recorded by Ilorin ${ }^{[4]} 71 \%$ of higher parity women had primary or secondary education 
only. This indicates that more the number of deliveries, the less money for education and training of children, so the vicious cycle continues. Those with higher educational qualifications were less likely to be grandmultiparous. Hence, female education is the key in making informed choices about available reproductive health care services and reduction of grandmultiparity. Among antepartum maternal complications, anaemia was present significantly in both groups; slightly higher in higher parity $25.9 \%$ versus $22 \%$, but the difference was not significant statistically. This finding is in keeping with findings of Aragaw etal ${ }^{[5]}$, Ramesh et $\mathrm{al}^{[6]}$, F Majoko et $\mathrm{al}^{[7]}$, Yahya etal ${ }^{[8]}$. Hypertensive disorders showed a statistically significant higher incidence among higher parity women. Gestational Diabetes mellitus and overt Diabetes was paradoxically reported less in higher parity women; in contrast to what was described in Wanda $\mathrm{K}$ et $\mathrm{al}^{[9]}$. This could be explained by higher obesity and other socioeconomic factors in lower parity women. Preterm delivery and twin pregnancy was reported less in higher parity as against former teaching. Increasing parity had no effect on malpresentation; in contrast to findings of Mgyaya A H etal ${ }^{[10]}$. IUGR was found to be higher in higher parity as against lower parity, but difference was not statistically significant. This contrasted with findings of Majoko $\mathrm{FM}^{[7]}$ Atonic PPH is supposed to be associated with increasing parity and the uterus fails to respond to oxytocics leading to life threatening situations .In the study, however, atonic PPH incidence was lower in higher parity. This is in keeping with Mgaya $\mathrm{AH}$ et $\mathrm{al}^{[10]}$ and against Aragaw et $\mathrm{al}^{[5]}$. Induction rate was more in lower parity. This shows that higher parity women more often went into spontaneous labour and rarely required inducton of labour. Second degree perineal injuries was recorded more in higher parity; in keeping with Aragaw et al ${ }^{[5]}$. Considering mode of delivery, $66.4 \%$ of lower parity and $72.5 \%$ of higher parity had normal vaginal delivery. The difference was not significant statistically. In Mgaya $\mathrm{AH}^{[10]}$ high incidence of Caesarean section was reported in grand multipara owing to obstetrician avoiding difficult vaginal delivery for Caesarean section. Meconium stained amniotic fluid was found to be slightly more in higher parity (3.1\% versus $2.3 \%)$.

\section{Conclusion}

The study concludes that higher parity continues to be a high risk pregnancy and is associated with increased maternal and perinatal complications. In general, in a low health resource setting, all pregnancies are prone to adverse outcomes; so adequate management of labour, a good referral system as well as practice of basic and comprehensive obstetric emergency care is mandatory. Higher parity women suffer from anemia, hypertensive disorder of pregnancy, IUGR babies, meconium aspiration and genital injury. Increasing awareness in community and institution of family planning services and provision of quality intrapartum and post partum care might improve maternal and perinatal outcome.

\section{Limitation of the Study}

Several confounding factors have not been addressed in the study, for instance nutritional status of woman, inter pregnancy interval. Because of the crossectional nature of the study, it fails to establish any casual influences on the low prevalence of uterine atony or Diabetes mellitus in the study. Hence, caution should be exercised in translating results based on outcomes to general population.

\section{Acknowledgements}

We thank the department of Obstetrics and Gynaecology, Government Medical College Manjeri and colleagues who had wholeheartedly given their support for the study. We wish to thank the Superintendent of Government Medical College Manjeri for allowing us to refer the labour records of the women who took part in the study. We also thank Dr Oommen Thomas for the statistical help.

\section{Conflicts of interest}

The authors declare that there are no conflicts of interest among the authors regarding publication of this paper. 


\section{Funding}

The authors received no financial support for research, authorship and/or publication of this article.

\section{References}

1. Charles Obinna Njoku, Sylvester Etenikang Abeshi, Cajethan Ife Emechebe. Grand Multiparity: Obstetric Outcome in Comparison with Multiparous Women in a Developing Country. OJOG> Vol.7 No.7, July 2017PP. 707-718

2. Idrisa, A. and Nwobodo, E.I. (1998) The Problems of Grandmultipara as Seen at the University of Maiduguri Teaching Hospital, Nigeria. Nigerian Journal of Medicine, 7, 165-167.

3. Ogedengbe, O.K. and Ogunmokun, A.A. (2003) Grandmultiparity in Lagos, Nigeria. Nigerian Postgraduate Medical Journal, 23, 374-377.

4. Omokanye, L.O. (2012) Obstetric Outcome of Grandmultiparous Women in Ilorin, Nigeria: A Five Year Review. Nigerian Journal of Health Sciences, 12, 16-19. http://www.nigeran-jhs.org/

5. Yesuf Ahmed Aragaw ${ }^{*}$ Mintesnot Mahtemsilllasie and Habtamu Jarso Grand Multiparity and Pregnancy Related Complications among Women Who Gave Birth at Jimma University Specialized Hospital, Jimma, Southwest Ethiopia. Gynecol Obstet (Sunnyvale) 7:438. doi: 10.4172/2161-0932.1000438

6. Ramesh BH, Praveen S Patil, Jennifer Joseph. Multigravidity a Major Risk Factor of Anaemia in Pregnancy and its Comparison in Primigravida Women in Raichur. National Journal of Laboratory Medicine. 2017 Oct, Vol-6(4): PO22-PO2
7. Majoko FM, Nyström L, Munjanja SP, Mason E, Lindmark G. Relation of parity to pregnancy outcome in a rural community in Zimbabwe. Afr J Reprod Health. 2004 Dec;8(3):198-206.

8. Yahya M Al Farsi Email author, Daniel R Brooks ${ }^{\dagger}$,Martha M Werler ${ }^{\dagger}$, Howar d J Cabral ${ }^{\dagger}$,Mohammed A Al-Shafei ${ }^{\dagger}$ and Henk C Wallenburg ${ }^{\dagger}$ Effect of high parity on occurrence of anemia in pregnancy: a cohort study BMC Pregnancy and Childbirth201111:7

9. Wanda K. Nicholson, MD, MPH, MBA12, Keiko Asao, MD, PHD3, Frederick Brancati, MD, MHS345, Josef Coresh, MD, PHD35, James S. Pankow, PHD6 and Neil R. Powe, MD, MPH, MBA345. The Atherosclerosis Risk in Communities study. Diabetes Care 2006 Nov; 29(11): 2349-2354. 10. Mgaya AH, Massawe SN, Kidanto HL, Mgaya HN. Grand multiparity: is it still a risk in pregnancy? BMC Pregnancy Childbirth. 2013 Dec 23;13:241. doi: 10.1186/1471-2393-13-241. 